Proceedings of the International School and Conference on Optics and Optical Materials, ISCOM07, Belgrade, Serbia, September 3-7, 2007

\title{
Laser Sheet Scattering and the Cameras' Positions in Particle Image Velocimetry
}

\author{
J. Ilić $\dot{c}^{a, *}$, DJ. ČAnTrAK ${ }^{a}$ AND M. SREĆKOVIĆ ${ }^{b}$ \\ ${ }^{a}$ Faculty of Mechanical Engineering, University of Belgrade, Serbia \\ ${ }^{b}$ Faculty of Electrical Engineering, University of Belgrade, Serbia \\ Simulations of laser sheet scattering by microparticles, based on the \\ generalized Lorenz-Mie theory for the case of numerous random spatial dis- \\ tributions of scattering particles, were done, using the novel computational \\ time saving strategy. This type of scattering by particles immersed in a fluid \\ flow and its recording on cameras, presents the essence of particle image \\ velocimetry systems. The continuous and large change of the intensity of a \\ scattered light falling on the camera causes the sequences of images of vary- \\ ing quality, which makes many of them useless. This paper shows how the \\ problem could be alleviated by determining the angles of low relative stan- \\ dard deviation of scattered light intensity and using them for recording, as \\ well as by avoiding the angles of high relative standard deviation of scattered \\ light intensity.
}

PACS numbers: 42.25Fx, 42.62.Eh, 42.68.Mj, 42.79.Qx

\section{Introduction}

With respect to the traditional point based techniques such as laser Doppler anemometry (LDA) and phase Doppler anemometry (PDA), particle image velocimetry (PIV), as a planar technique, has significantly reduced data acquisition time [1]. On the other hand, it lacks some of the information about temporal velocity characteristics which are especially important in high swirling flows [2]. It could be overcome by recording the sequences of flow field images. That is considerably accomplished in two-component PIV systems $-2 \mathrm{C}-2 \mathrm{D}$, recording only in-plane components. In order to obtain the reliable out of plane component data, and even 3D data, several methods are evolving. The stereoscopic PIV with two off axes cameras is among them [1]. Most of the efforts put in improving the digital PIV have considered processing techniques for extracting the particle displacement and its validation [3]. Acknowledging their vital importance, we would like to draw attention to the following, though less frustrating, problem. During the recording of a sequence, with camera optical parameters set according to expected overall irradiance, the brightness of images varies rapidly and unpredictably. This is the reason why, in stereoscopic PIV systems, at the best, many of the images in a

${ }^{*}$ corresponding author; e-mail: jilic@mas.bg.ac.yu 
sequence are of good quality but rarely all of them are of good quality. Our idea was to find out the explanation of this phenomenon and if it could be alleviated by adequately chosen camera positions (angles).

To address this problem, laser sheet scattering by spheres has been considered (very often, standard tracer particles are spheres). Lorenz-Mie theory, the exact solution of the electric field of light scattered by dielectric spheres, is valid only for plane wave illumination. Therefore, generalized Lorenz-Mie theory (GLMT) should be applied [4]. Though widely used in radiation pressure calculations for optical trapping [5], and applied in simulations of LDA and PDA [6], as far as we are aware of, there are very few reports of GLMT based simulations of laser sheet scattering $[7,8]$.

The rigorous analytical solution of the scattering by assemblies of spheres [9] would require extremely robust calculation program even for the case of two spheres. In order to scrutinize the phenomenon, being aware of the simplification, only the scattering that contributes the most to the total scattered intensity has been considered. It is the scattering of the direct laser sheet. The scattering of light scattered by other particles has been neglected. Besides, two cases have been analyzed: when there was (case (I)) and when there was not (case (II)) the interference of the light scattered from different particles.

This paper reports the GLMT based simulation of scattering by 500 to 4000 particles. It proves that camera position does matter, and proposes the method of finding out more suitable positions.

\section{Experimental setup}

The experimental setup of the PIV system considered by the model presented in this paper is shown in Fig. 1a. The fluid flow, that was investigated, is confined in horizontal transparent cylindrical tube. Spherical tracers - particles (diameter $0.6 \mu \mathrm{m}$, refractive index of 1.47) are provided by calibrated atomizer and "sucked in" by the axial fan at the pipe inlet. Laser sheet $(\lambda=532 \mathrm{~nm})$, performing in pulsed mode, illuminates the particles and undergoes scattering by them. Pulses, fired with the known separation time (usually $0.01-0.5 \mathrm{~ms}$ ), are synchronized with the cameras' recordings by PC controlled synchronizer. The recorded images are used to calculate the displacements of tracer particles and according to them the flow velocity field is deduced.

\section{Model}

Model is based considering Cartesian system $(O-x y z)$ presented in Fig. 1. Let us note that, in Figs. 1a-c, three different views of the same Cartesian system are presented. In order to get better perspective view of the angles of interest, the Cartesian system in Fig. 1a is rotated (first $90^{\circ}$ clockwise around the $x$ axes then $90^{\circ}$ counter-clockwise around the $z$ axes) and presented in Figs. 1b and c. Though Fig. 1a shows two definite positions of the cameras, in the hereafter presented 


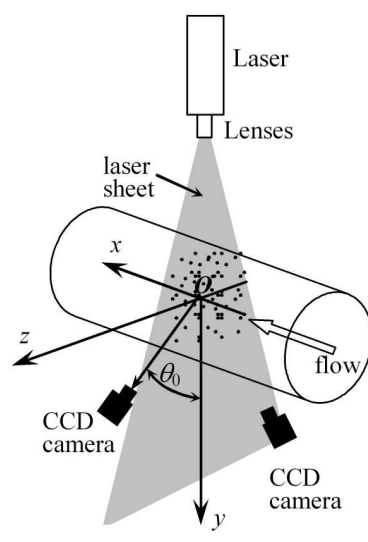

(a)

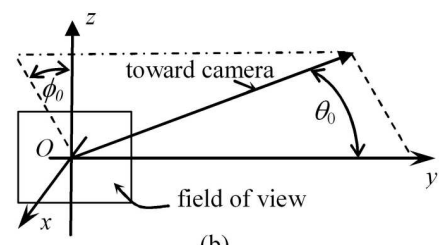

(b)

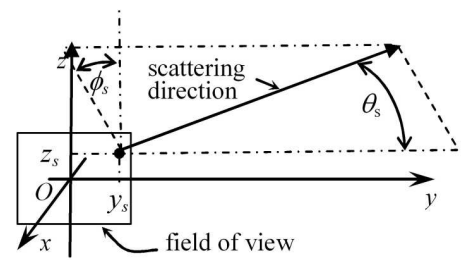

(c)

Fig. 1. (a) The core of PIV experimental setup along with the Cartesian system used in the model; (b) definition of angles that determine the camera position $\theta_{0}$ and $\phi_{0}$; (c) definition of scattering $\theta_{\mathrm{s}}$ and azimuth $\phi_{\mathrm{s}}$ angle in a particle frame.

model, arbitrary position of the camera is considered. Origin $O$ is at the center of the camera's field of view (in this simulation $16 \times 12 \mathrm{~cm}$ ). Laser sheet, propagating toward positive $y$ direction and linearly polarized in $z$ direction, is being scattered by particles randomly distributed within the field of view. The camera's position is defined by angles $\theta_{0}$ and $\phi_{0}$ (Fig. $1 \mathrm{~b}$ ) and the distance $R_{0}$ (here $0.5 \mathrm{~m}$ ) between $O$ and the camera (not presented in Fig. 1). The position of any scattering particle $\left(y_{\mathrm{s}}, z_{\mathrm{s}}\right)$ determines its own scattering and azimuth angle $\theta_{\mathrm{s}}$ and $\phi_{\mathrm{s}}$ (Fig. 1c). They are related to the camera position parameters, by the derived expressions

$$
\begin{aligned}
\tan \theta_{\mathrm{s}} & =\frac{\left(R_{0}^{2} \sin ^{2} \theta_{0}+z^{2}-2 z R_{0} \sin \theta_{0} \cos \phi_{0}\right)^{1 / 2}}{R_{0} \cos \theta_{0}-y}, \\
\sin \phi_{\mathrm{s}} & =\frac{R_{0} \sin \theta_{0} \sin \phi_{0}}{\left(R_{0} \cos \theta_{0}-y\right) \tan \theta_{\mathrm{s}}} .
\end{aligned}
$$

Complex amplitude functions of parallel $\left(S_{\mathrm{Ps}}\right)$ and perpendicular $\left(S_{\mathrm{Ns}}\right)$ polarization component of the light scattered on sphere $s$, have been calculated according to generalized Lorenz-Mie theory [10]. They present double sums of terms depending on particle size and refractive index $\left(a_{\mathrm{ns}}, b_{\mathrm{ns}}\right)$, particle position within the laser sheet and waist radii (beam shape coefficients $g_{n \mathrm{TMs}}^{m}, g_{n \mathrm{TEs}}^{m}$ ), and on scattering angle $\theta_{\mathrm{s}}$ (Legendre functions $\pi_{n \mathrm{~s}}^{|m|}, \tau_{n \mathrm{~s}}^{|m|}$ ). $a_{\mathrm{ns}}, b_{\mathrm{ns}}$ involve Bessel functions. $g_{n \mathrm{TMs}}^{m}, g_{n \mathrm{TEs}}^{m}$ present complex functions involving triple sums. $\pi_{n \mathrm{~s}}^{|m|}, \tau_{n \mathrm{~s}}^{|m|}$ also depend on particle position.

(I) If the coherence length of laser sheet is longer than field of view dimensions, the electric field intensity of laser sheet scattered by an assembly of $N$ spherical particles is the result of superposition of electric fields scattered by each of the particles, i.e., 


$$
E=\sum_{s=1}^{N} \boldsymbol{E}_{s}=\frac{\lambda}{2 \pi}\left|\boldsymbol{E}_{0}\right|\left(\sum_{s=1}^{N}\left|S_{\mathrm{P} s}\right| \frac{\cos \varphi_{s}}{R_{s}} \boldsymbol{e}_{\theta}+\sum_{s=1}^{N}\left|S_{\mathrm{N} s}\right| \frac{\sin \varphi_{s}}{R_{s}} \boldsymbol{e}_{\varphi}\right),
$$

where $\lambda$ is the laser wavelength, $E_{0}$ is the field intensity on the laser sheet axes and $R_{s}$ is the distance between the sphere $s$ and the recording camera (CCD chip). The directions of the electric field vectors scattered from different particles are very close but not exactly the same as it is supposed in Eq. (2). The real value is slightly, but certainly not much, smaller.

(II) In the opposite case when none of the scattered light is coherent, the total intensity is the sum of single particles scattered intensities. (For high particle concentration it is hardly true even for the Sun light.)

Angular intensity distributions have been calculated for both cases - (I) and (II), for 20 different random particle distributions over the field of view. Then, mean intensity and its relative standard deviations (RSDs) are found, for each of the camera position angles $\theta_{0}$.

In order to save computational time, first, for the given particles and laser sheet dimensions, the maximum values of $n$ and $m$ for which $a_{n \mathrm{~s}}, b_{n \mathrm{~s}}$ and $g_{n \mathrm{TMs}}^{m}$, $g_{n \mathrm{TEs}}^{m}$ are greater than $10^{-15}$ are found. Then, terms with $m=0$ are grouped and separated from the others. Separately, for $m>0$ and for $m<0$, the beam shape coefficients $g_{n \mathrm{TMs}}^{m}, g_{n \mathrm{TEs}}^{m}$ and $g_{n \mathrm{TMs}}^{m}, g_{n \mathrm{TEs}}^{m}$ are calculated, respectively. Therefore, the sums in Eq. (8) of Lock and Gouesbet [11] could be transformed in considerably shorter sums: $\sum_{n=1}^{\infty} \sum_{m=-n}^{n} \rightarrow \sum_{n=1}^{n_{\max }}{ }_{m=0}+\sum_{m=1}^{m_{\max }} \sum_{n=m}^{n_{\max }}$. Complex amplitude functions then read as

$$
\begin{aligned}
& S_{\left(\begin{array}{l}
N \\
\mathrm{P}
\end{array}\right) \mathrm{s}}=\sum_{n=1}^{n_{\max }} \frac{2 n+1}{n(n+1)}\left(\begin{array}{c}
\mathrm{i} g_{n \mathrm{TEs}}^{0} b_{n \mathrm{~s}} \\
g_{n \mathrm{TMs}}^{0} a_{n \mathrm{~s}}
\end{array}\right) \tau_{n \mathrm{~s}}^{0}+\sum_{m=1}^{m_{\max }} \exp \left(\mathrm{i} m \phi_{\mathrm{s}}\right) \sum_{n=m}^{n_{\max }} \frac{2 n+1}{n(n+1)} \\
& \quad \times\left[\left(\begin{array}{c}
m \pi_{n \mathrm{~s}}^{m} \\
\tau_{n \mathrm{~s}}^{m}
\end{array}\right) a_{n \mathrm{~s}}\left(g_{n \mathrm{TMs}}^{m} \mp g_{n \mathrm{TM}}^{m}\right)+\left(\begin{array}{c}
\tau_{n \mathrm{~s}}^{m} \\
m \pi_{n \mathrm{~s}}^{m}
\end{array}\right) \mathrm{i} b_{n \mathrm{~s}}\left(g_{n \mathrm{TEs}}^{m} \pm g_{n \mathrm{TEs}}^{m}\right)\right] .
\end{aligned}
$$

\section{Results and discussion}

The results obtained in the cases of three chosen numbers of spherical particles are presented in Fig. 2 for the case (I) and in Fig. 3 for case (II). Only the results of the calculation for the case $\phi_{0}=\pi / 2$ are reported in this paper.

According to Fig. 2, for all directions (except in-line) RSDs are very high - up to $150 \%$, and almost never less than $50 \%$, which explains highly varying brightness in a sequence of PIV images, if coherent light is involved. On the other hand, RSDs are much smaller in the case of low coherence light, ranging from $1 \%$ up to $6 \%$ (Fig. 3). Also, in both cases, RSDs are smaller for larger particle concentrations for angles between $20^{\circ}$ and $160^{\circ}$.

Furthermore, the directions that are not suitable for recording are following: (1) In-line recordings - at $0^{\circ}$ and $180^{\circ}$, since there are, in both cases, the highest RSDs; they are omitted in Fig. 3 for being two orders of magnitude larger than the all others. (2) RSDs considerably oscillate with the direction angle $\theta_{0}$ in the 


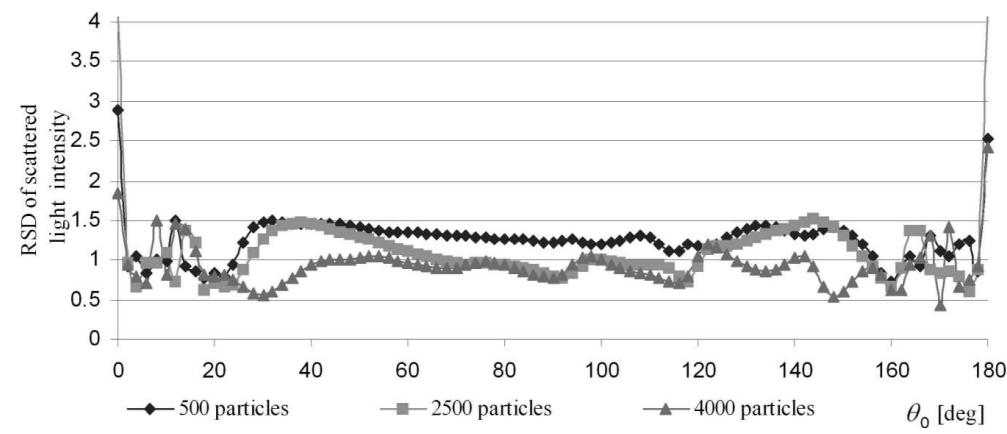

Fig. 2. RSD of the intensity of the laser sheet light scattered by 500 particles (diamonds), 2500 particles (squares) and 4000 particles (triangles), versus the camera position angle $\theta_{0}$, in the case (I) i.e. when a coherence length is longer than field of view dimensions, thus the scattered electric field is the result of superposition of electric fields scattered by each of the particles.

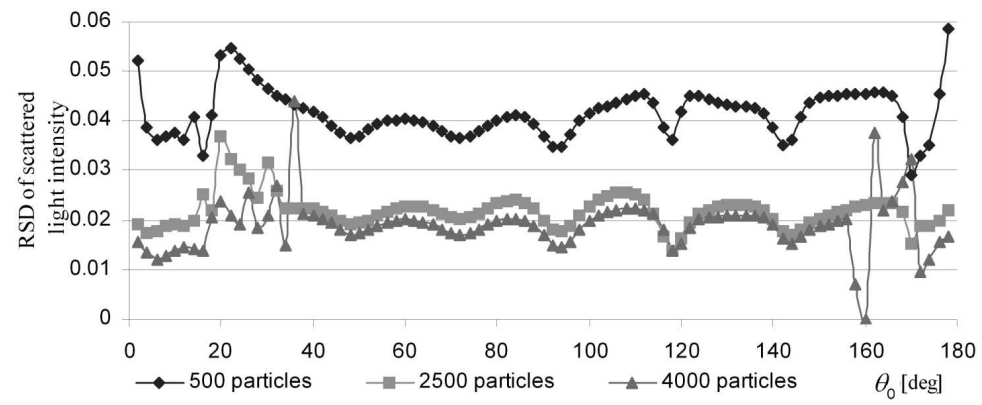

Fig. 3. RSD of the intensity of the laser sheet light scattered by 500 particles (diamonds), 2500 particles (squares) and 4000 particles (triangles), versus the camera position angle $\theta_{0}$, in the case (II) i.e. low coherence case, where none of the scattered light is coherent, thus the total intensity is the sum of single particles scattered intensities.

regions close to in-line (forward and backscattering): in the case (I) for angles $0^{\circ}-20^{\circ}$ and $160^{\circ}-180^{\circ}$, and in case (II) for angles $10^{\circ}-40^{\circ}$ and $155^{\circ}-180^{\circ}$.

Finally, the directions that are suitable for recording are those of local RSD minima: (1) in both cases around $90^{\circ}$ (except for high coherence lowest concentration); moreover, in that direction total intensity and, consequently, the brightness is less varying not only with the particle spatial distribution but also with the particle concentration; that adds to the explanations of the success of $2 \mathrm{D}$ measurements, which use recording in this direction; (2) the high coherence local minima of RSDs for all numbers of particles (also those not presented in Fig. 2) are around $20^{\circ}$ and around $160^{\circ}$, and there are several other local minima in the low coherence case (Fig. 3) - all suitable for stereoscopic PIV and other off-axes measurements. 
This paper considers two extreme cases: (a) when the coherence length of laser sheet light is larger than the field of view, and (b) when the coherence length of laser sheet light is smaller than the minimum distance between the particles. Intermediate case requires much more complicated model than the one developed in this paper. Also, here explained model could be readily extended for the case of polydisperse tracer particles.

Nevertheless, this model does explain the varying quality of PIV images in a sequence. It, also, illustrates the method of finding the suitable and not suitable directions that can be applied to any other laser sheet and tracer particle parameters. By recording in suitable and avoiding not suitable directions, higher percent of images in a sequence would be useful for fluid flow analyses without any additional processing. In this way, apart from providing more information about the fluid flow, processing procedures are simplified and processing time is saved.

\section{Acknowledgments}

The Government of the Republic of Serbia and the Ministry of Science and Environmental Protection, have provided the fund for capital equipment investments in science, including this PIV system, as a part of the National Investment Program in 2006/2007. We are grateful for their support of great significance.

\section{References}

[1] R.J. Adrian, Exp. Fluids 39, 159 (2005).

[2] S. Čantrak, M. Benišek, M. Nedeljković, in: Spatio-temporal Structure and Chaos in Heat and Mass Transfer Process, Eds. L.M. Pismen, M.S. Todorović, Mrlješ \& Sons Ltd., Belgrade 1993, p. 29.

[3] F. Scarano, Meas. Sci. Technol. 13, R1 (2002).

[4] K.F. Ren, G. Gouesbet, G. Grehan, Appl. Opt. 37, 4218 (1998).

[5] J.A. Lock, Appl. Opt. 43, 2532 (2004).

[6] H. Qiu, C.T. Hsu, Appl. Opt. 38, 2737 (1999).

[7] J.A. Guerrero-Viramontes, D. Moreno-Hernandez, F. Mendoza-Santoyo, M. Funes-Gallanzi, Meas. Sci. Technol. 17, 2328 (2006).

[8] P. Padilla Sosa, J.E. Valdez, L.R. Berriel, L.R. Sahagun Ortiz, M. Funes-Gallanzi, Opt. Eng. 42, 459 (2003).

[9] G. Gouesbet, G. Grehan, J. Opt. A, Pure Appl. Opt. 1, 706 (1999).

[10] K.F. Ren, G. Grehan, G. Gouesbet, J. Opt. Soc. Am. A 11, 2072 (1994).

[11] J.A. Lock, G. Gouesbet, J. Opt. Soc. Am. A 11, 2503 (1994). 